LAPP-EXP 2002-03

\title{
The ATLAS liquid argon calorimetry system
}

\author{
Pascal PERRODO \\ LAPP, IN2P3-CNRS, \\ 9, chemin de Bellevue, BP110, \\ F-74941, Annecy-le-Vieux cedex
}

\begin{abstract}
The status of construction and recent test beam results for the ATLAS liquid argon calorimetry system (electromagnetic, hadronic and forward calorimeters) are reported as well as a new development on the first-level trigger system for electromagnetic clusters and isolated hadrons.
\end{abstract}

Talk given at the $31^{\text {th }}$ International Conference for High Energy Physics,

24-31 july 2002, Amsterdam, The Netherlands 


\title{
The ATLAS liquid argon calorimetry system
}

\author{
Pascal Perrodo ${ }^{\text {** }}$ \\ ${ }^{a}$ LAPP, 9 chemin de Bellevue, BP110, 74941 Annecy-le-vieux CEDEX, FRANCE
}

The status of construction and recent test beam results for the ATLAS liquid argon calorimetry system (electromagnetic, hadronic and forward calorimeters) are reported as well as a new development on the first-level trigger system for electromagnetic clusters and isolated hadrons.

\section{Introduction}

The ATLAS detector is a multi-purpose detector which will be installed on the LHC collider at CERN. It has been designed to search for possible new physics phenomena in the energy range of $\approx 100 \mathrm{GeV}$ to $\approx 1 \mathrm{TeV}$. The traditional signatures for new physics are based on high $P_{T}$ photons, leptons $\left(e^{ \pm}, \mu^{ \pm}, \tau^{ \pm}\right)$and missing transverse energy. The calorimeters plays a crucial role in such a search.

The choice of liquid argon calorimetry has been made for its radiation hardness, stability and good calorimetry performances. A short description is given here pointing out the specificities of each detector and the evolution of the design with respect to the initial one [1]. Recent test beam results of series modules for the electromagnetic (EM), hadronic (HEC) and forward calorimeters (FCAL) are presented (more details can be found in [2]) as well as the status of the construction.

The calorimeter analog signals are used to build not only the first level trigger signal on jets, total and missing transverse energies but also to trigger on events with isolated electrons/photons or isolation hadrons (likely coming from hadronic $\tau^{ \pm}$ decays) which are crucial for the LHC physics. A new development of a board to implement this trigger is presented.

*on behalf of the ATLAS liquid argon and first-level trigger groups

\section{The electromagnetic calorimeter}

The EM calorimeters are required to measure the energy and position of $e^{ \pm}$and $\gamma$ with a good uniformity as well as a good $\gamma / \pi^{0}$ separation. The response has to be fast, and an accordion shaped geometry for lead absorbers and electrodes has been chosen which also insures a total hermiticity in $\phi$. Lots of care has been taken at the start of the production period with the honeycomb spacers to avoid high voltage shorts, with the bending of the kapton-copper electrodes, and the handling of the resistive ink which reduces the impact of sparks on electronics.

The ionisation signals are amplified and $\mathrm{CR}-\mathrm{RC}^{2}$ shaped, then sampled every $25 \mathrm{~ns}$ before digitization. These samples are used after first level trigger decision to reconstruct the energy and the arrival time for each channel using optimal filtering techniques. The calibration is achieved by injecting exponentially decaying signals with very well known amplitudes through precision $(0.1 \%)$ resistors in the $\ell$ Ar. It has been observed recently that these resistors are sentitive to possible $\mathrm{HV}$ sparks. A protection diode has therefore been installed on each channel.

The EM calorimeters (barrel and endcap) are currently half produced and assembled. The insertion of this first half into the cryostats should occur beginning of 2003 .

Seven series modules have been tested with a beam at CERN. A substantial effort has been made to understand the shape and the normalisation of the calibration and physics signals with respect to the electrical characteristics of the detec- 


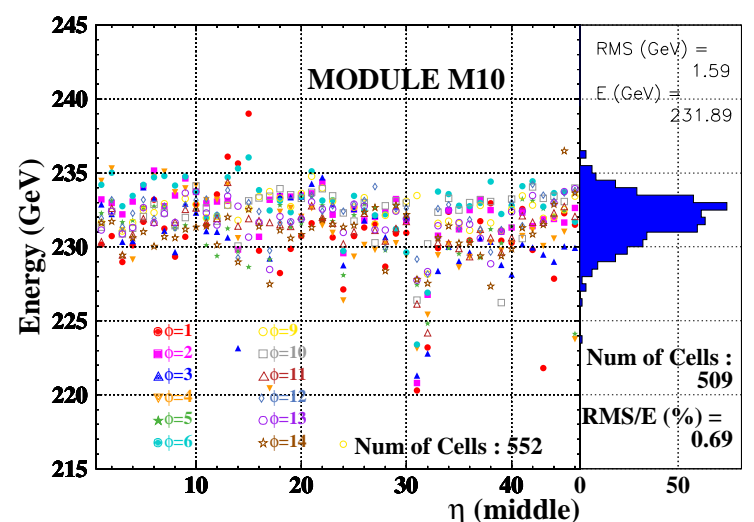

Figure 1. Uniformity of energy response for one electromagnetic barrel module (M10).

tor (inductances, ground returns and cross talk). This has led to an absolute electronic calibration and the possibility to compute meaningful calibrated optimal filtering coefficients [3]. From this the uniformity in position of the energy response has been extracted: over one full series module a promissing figure of $0.7 \%$ is obtained for the uniformity after clustering and time corrections (see Fig. 1) when $0.7 \%$ is expected over the whole EM calorimeter.

Two HV lines supply a region $\Delta \eta=0.2, \Delta \phi=$ 0.2 . When one is missing, energy has to be multiplied by 2 . However at the boundary with a fully supplied region a non linear behaviour $(\approx 5 \%)$ has been observed. It has been fully reproduced by simulation and is now understood as a geometrical effect of the accordion. This proves that such a local possible HV problem can be fully corrected.

The position resolution has been studied for the endcap calorimeter. For the front and middle layers (granularities $\Delta \eta=0.025 / 6, \Delta \eta=$ $0.025)$ the resolution has a sampling terms of $(2.69 \pm 0.05) \mathrm{mm} / \sqrt{ } \mathrm{E}(\mathrm{E}$ in $\mathrm{GeV})$, and $(5.40 \pm$ $0.20) \mathrm{mm} / \sqrt{ } \mathrm{E}$ respectively. This can be converted into an angular resolution of $50 \mathrm{mrad} / \sqrt{ } \mathrm{E}$ in agreement with the ATLAS requirements. Similarly the position resolution can also be measured from the EM barrel middle compartment for various beam energies. A $3.4 \mathrm{~mm} / \sqrt{ } \mathrm{E}$ sampling term with $0.32 \mathrm{~mm}$ constant term is extracted at $\eta=0.47$.

Finally preliminary results on $\gamma / \pi^{0}$ separation have been obtained using events recorded from a photon beam. After kinematical $\gamma$ pairing and cuts using the first strip compartment a promissing rejection factor (due to the first calorimeter compartment with fine $\Delta \eta=0.025 / 8$ segmentation) between 2.6 and 3 (as a function of the photon's $P_{T}$ ) is obtained for a $90 \%$ photon's efficiency when the figure of 3.0 is expected especially for the $\mathrm{H} \rightarrow \gamma \gamma$ search.

\section{The Hadronic Endcap Calorimeter}

The HEC calorimeter is designed for forward jet measurements in association with the EM endcap calorimeter. It requires a fast response in a high radiation environment. It consists of flat copper absorber plates interleaved with electrodes organised on the principle of the electrostatic transformer (EST). This increases the signal amplitude without increasing the capacitance and the required $\mathrm{HV}$. The readout is achieved with cryogenics AsGa preamplifiers in order to optimize the signal-to-noise ratio, followed in the warm by pre-shapers instead of preamplifiers like for the EM and then the same chain as for EM. The calibration is performed in the cold in a similar way as the EM. Currently $90 \%$ of the HEC calorimeters modules are produced and tested. A first wheel of 32 modules is completed (see Fig. 2).

Beam tests have been performed on 24 over 134 HEC modules at CERN. First of all the calibration and physics signals have been simulated according to an electrical model of the readout circuits. The agreement found is within $1 \%$. Measurements performed at various $\ell$ Ar temperatures have been used to extract the ionisation drift time from the physics pulse shape and the relative amplitude variation after shaping is found to be $2 \% /{ }^{\circ} \mathrm{K}$ in agreement with ionisation models and similar measurements performed with the EM. The drift velocity and the ionisation cur- 


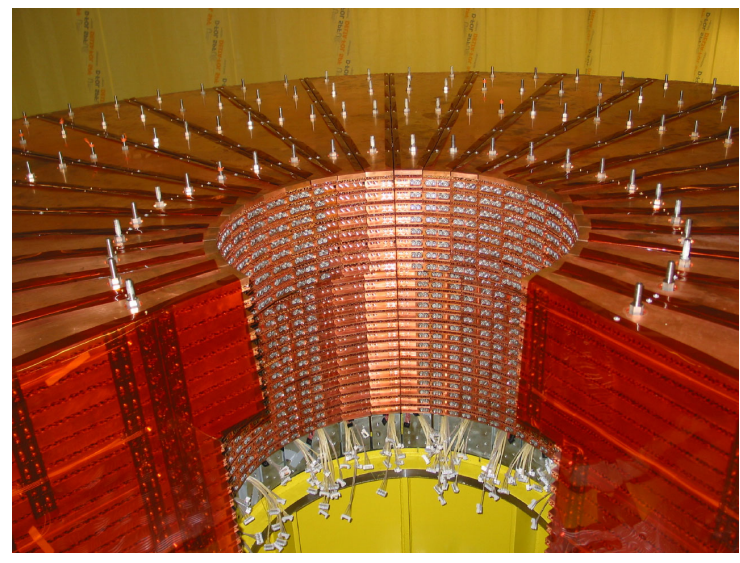

Figure 2. View of the first endcap wheels (almost) assembled.

rent have also been measured as a function of the high voltage in the range $[1,10] \mathrm{kV} / \mathrm{cm}$ and are in good agreement with the models. The modules have been scanned in 15 points with $e^{ \pm}$and $\pi^{ \pm}$beams. The sampling and constant terms for electrons are found to be $(21.4 \pm 0.2) \%$ and $(0.3 \pm 0.2) \%$ with a spatial uniformity better than $1 \%$. These two terms have been checked to be stable in position over the calorimeter within the errors and are compatible with the simulation expectations. The $\mu^{ \pm}$to noise ratio has been measured to be $\approx 5$ and the $e / \mu$ ratio is found to be $0.93 \pm 0.04$ in agreement with simulations. Finally the $\pi^{ \pm}$resolutions as a function of the beam energy give sampling and constant terms estimated to be $(70.6 \pm 1.5) \%$ and $(5.8 \pm 0.2) \%$ with an intrinsic ratio $e / h=1.49 \pm 0.10$ in fair agreement with the GCALOR simulation program.

\section{The Forward Calorimeter}

The FCAL calorimeter extends the detector acceptance from $|\eta|=3.2$ to 4.9 . It plays an important role for forward jet tagging, vital for essential physics channels at LHC [5]. It has to cope with a constant high particle flux from minimum bias events and a very high radiation level. The technological choice consists of successive wheels in copper then tungsten for the absorber. The sensitive volumes are thin cylindrical $\ell$ Ar gaps (tube/rod assemblies). A consequence is a fast drift time and a large cell capacitance. In order to adapt the signal to the same readout electronics than the EM an 2:1 autotransformer is used. Currently one half of the FCAL detector is completed and being assembled at CERN, the other half is expected for june 2003 .

FCAL prototype modules have been tested in a beam at CERN in 1998. Energy resolution and linearity for $e^{ \pm}$and $\pi^{ \pm}$beams as well as the $\mu^{ \pm}$to noise ratio have been studied [6]. The $e^{ \pm}$ linearity is measured to be within $1 \%$. For $\pi^{ \pm}$ beam the possibility to apply weights with a radial dependence has been studied and it has been shown that the noise can be improved by $\approx 10 \%$ and the sampling term reduced from $89 \% / \sqrt{ } E$ to $80 \% / \sqrt{ } E$. The radial dependence of the weights shows well the suppression of the central part, mainly due to $\pi^{0}$ 's, the enhancement of the peripheral zone and the suppression at large radius corresponding to the presence of coherent noise. This weighting method looks interesting for the FCAL calorimeter as the forward jets are very narrow. So the lateral shower extension for jets is mainly governed by the hadronic shower phenomenon and not by the jet opening.

\section{Isolated clusters at first level trigger}

Triggering at first level on isolated electromagnetic showers $\left(e^{ \pm}, \gamma\right)$ and isolated hadrons most likely coming from $\tau^{ \pm}$is crucial for LHC physics as these particles are among the main signatures for possible new phenomena.

The calorimeter-based first-level trigger signals are formed from the analog signals after shaping. They are aligned in time, summed into trigger towers $(\Delta \eta=0.1, \Delta \phi=0.1)$, converted into transverse energy, digitized and associated to a beam crossing number [7]. These digitized signals are used to form jet and missing energy clusters or for isolated e.m./hadron clusters.

The isolated e.m./hadron cluster trigger is based on a sliding window algorithm using $4 \times$ 4 trigger towers in both electromagnetic and hadronic calorimeters. This window is divided 
into four regions: the e.m. core, the e.m isolation ring, the had. core and the had. isolation ring (see [7] p. 45). The e.m. trigger is achieved by requiring energy in two adjacent trigger towers in the e.m. core and no energy elsewhere. The isolated hadron trigger is on an other hand achieved by requiring energy in the the sum of two adjacent trigger towers in the e.m. core and all 4 trigger towers in the hadronic core, and no energy in both isolation rings.

The cluster trigger covers the phase space, $|\eta| \leq$ 2.5 , and all $\phi$. It consists of 4 crates, each containing 14 cluster Processing Modules (CPM). Each CPM (see Fig. 3) processes $4 \times 16$ windows using 8 FPGA's (Xilinx Virtex chip XCV1000E). Because of the slidling window algorithm a CPM needs information processed by the adjacent boards. This communication is achieved via the crate back plane using a $160 \mathrm{Mbs}^{-1}$ serial data stream in order to limit the number of electrical connections.

Simulations of this implementation have been made. It has been shown that the cluster algorithm itself is evaluated within 25ns. Including the other steps required, the cluster trigger takes a total of $175 \mathrm{~ns}$, to be compared with the total time available to the level-1 calorimeter trigger of about 600 ns. A complete CPM has now been produced and is under test.

\section{Conclusion}

The status on the construction of the ATLAS liquid argon calorimeters (barrel, endcap electromagnetic and hadronic and forward) has been reported. Almost half of the detector is up to now produced. Several test beam have been performed on the serie modules and give good results in agreement with expectations. On an other hand a board for triggering electromagnetic clusters and isolated hadrons at the first-level trigger has been developed. The performances in time are good within the acceptable first-level time window.

\section{Acknowledgements}

I am indebted to the members of the ATLAS collaboration, to P. Loch, P. Schacht, L. Serin and especially J. Garvey for his carefull reading of the manuscript.

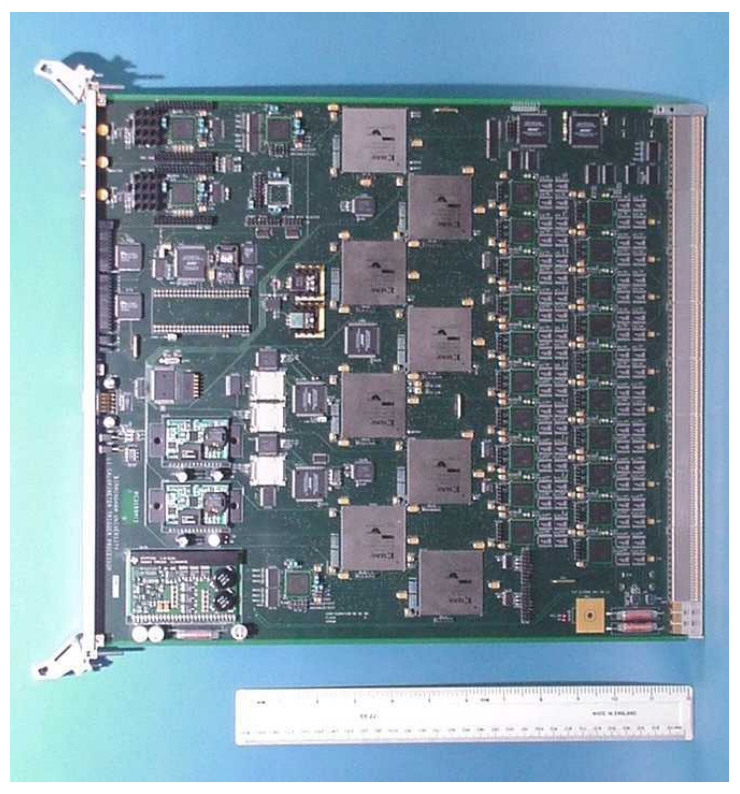

Figure 3. The CPM board for the electromagnetic and isolated hadron first-level trigger.

\section{REFERENCES}

1. ATLAS Liquid Argon Calorimeter Technical Design Report, CERN/LHCC/96-41.

2. International Conference on Calorimetry in High Energy Physics, Pasadena, March 25-29, 2002, see talks given by M. Fincke-Keeler, K.K. Joo, A. Kiryunin, S. Rodier and D. Zerwas.

3. ATLAS Coll., Performance of the barrel module 0 of the ATLAS electromagnetic calorimeter, to be published in NIM.

4. ATLAS Coll., Performance of the end-cap module 0 of the ATLAS electromagnetic calorimeter, to be published in NIM.

5. ATLAS Detector and Physics Performance, vol 2, CERN/LHCC/99-15.

6. A. Savine et al., in Proc. of the $7^{\text {th }}$ Int. Conf. on Calorimetry in High Energy Physics, Tucson $199 \%$.

7. ATLAS First-level Trigger Technical Design Report, CERN/LHCC/98-14. 